\title{
Analysis on the Inclination of Anchor Chain and Barrel in Mooring System
}

\author{
Zhihong Jin \\ North China Electric Power University, Bao Ding 071000, China \\ zijinzh@163.com
}

Keywords: mooring system, virtual work principle, principle of virtual displacement.

\begin{abstract}
Anchor chain of mooring system can consist of several steel pipes. A series of stress analysis is used in this paper and the steel pipe mechanics model is established. According to the principle of virtual displacement and virtual work principle, the sum of gravity, buoyancy and tensile force is zero, because all the constraints which are given by the steel pipe are ideal constraints. Inclination can be calculated by the steel pipe in the sea wind speed of $12 \mathrm{~m} / \mathrm{s}$ and $24 \mathrm{~m} / \mathrm{s}$ in Table 1 and Table 2. Finally, according to the equilibrium equation, the inclination of the barrel at sea speed of $12 \mathrm{~m} / \mathrm{s}$ and $24 \mathrm{~m} / \mathrm{s}$ is $1.83^{\circ}$ and $2.85^{\circ}$ respectively.
\end{abstract}

\section{Introduction}

The transmission nodes of the near shallow sea observation network are composed of buoy system, mooring system and underwater acoustic communication system. The mooring system consists of steel pipe, barrel, heavy ball, welding anchor chain and special anti-drag anchor composition. The end of the anchor chain and the anchor at the junction of the tangential direction and the seabed angle of can not be more than 16 degrees. Otherwise the anchor will be dragged, resulting in node shift is lost. The underwater acoustic communication system is installed in the sealed cylindrical barrel, and the barrel is connected with the fourth steel pipe and the welding anchor chain. [1]

When the barrel is vertical, the sound of the acoustic communication equipment works best. If the barrel tilted, the work of the equipment will be affected. When the inclination angle of the barrel (the angle between the barrel and the vertical line) exceeds 5 degrees, the working efficiency of the equipment is poor. In order to control the inclination angle of the barrel, the barrel and the anchor chain link can hang a heavy ball, so that the inclination angle of the steel drum as small as possible.

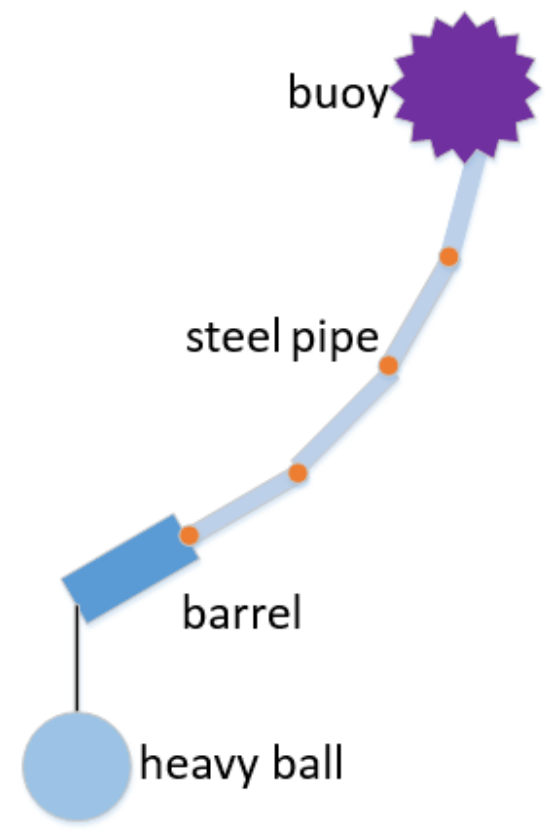

Fig1. The mooring system 


\section{The steel pipe and the barrel mechanics model}

\section{Assumptions.}

(1) In order to simplify the subsequent numerical calculation process, assume that the ball and steel are steel material.

(2) The wind of the sea will inevitably affect the flow of seawater, which will lead to changes in the force of the steel pipe and the barrel. However, in this paper, it is assumed that the wind speed direction is parallel to the sea level and the wind speed does not affect the sea surface water flow.

\section{Analysis of steel pipes force.}

In seawater under static conditions, the effect of sea water on the mooring system in the horizontal direction will be the only factor that causes the change of the sea buoy position, and the whole mooring system will move. When a certain period of time after the wind, we can think that the entire mooring system has been in a state of balance, select the first section of the steel pipe and buoy connection for the coordinates of the origin, the establishment of plane Cartesian coordinates, as shown in Fig 2.

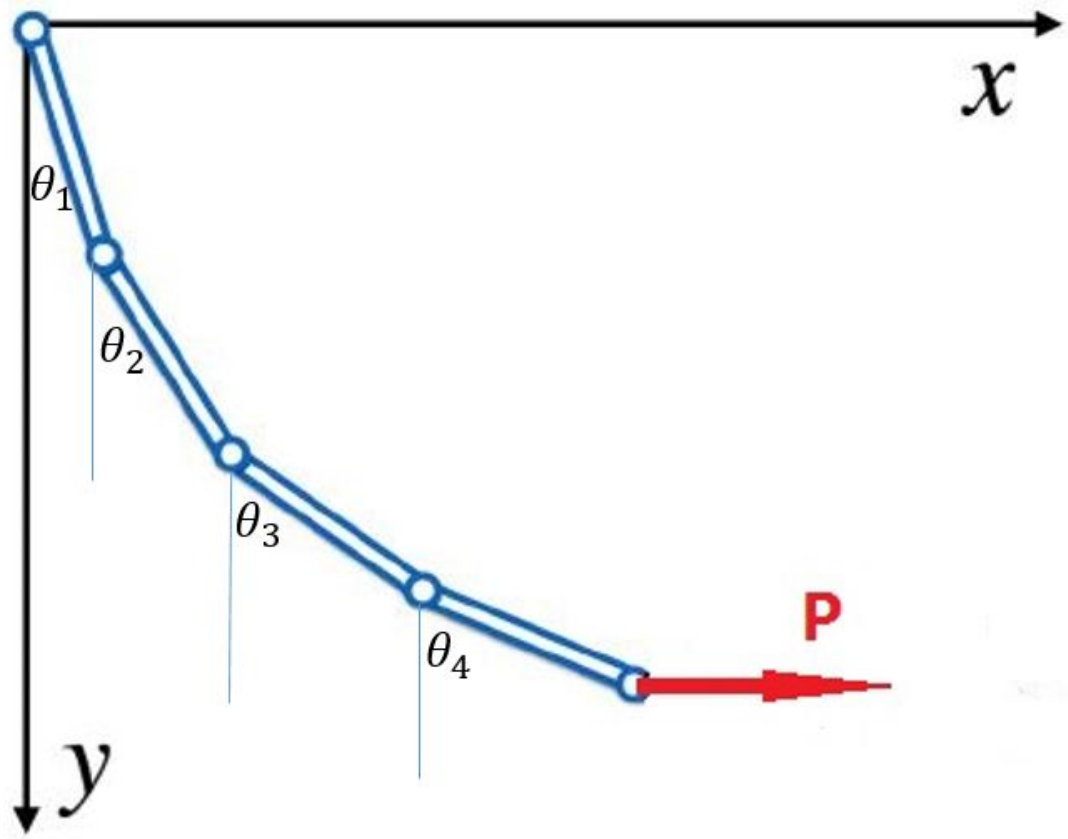

Fig 2. Free-body diagram of steel pipes

The center of mass of the i-th pipe[2] are

$$
\begin{aligned}
& x_{i}=a \sum_{\mathrm{j}=1}^{i-1} \sin \theta_{j}+a \sin \theta_{i} / 2(1.1) \\
& \mathrm{y}_{i}=a \sum_{j=1}^{i-1} \cos \theta_{j}+a \cos \theta_{i} / 2(1.2)
\end{aligned}
$$

Where

a indicates the length of the pipe

$\theta_{i}$ denotes the angle between the i-th steel pipe and the vertical direction.

$\theta_{j}$ denotes the angle between the j-th steel pipe and the vertical direction.

According to the theory of virtual displacement[3] in analytical mechanics and the principle of virtual work[4], the power of generating virtual work is: gravity, buoyancy and main force.

The virtual work of the steel pipe gravity is

$$
\delta W_{G}=-m g a \sum_{i=1}^{n}\left(\sum_{j=1}^{i-1} \sin \theta_{j} \delta \theta_{j}+\sin \theta_{i} \delta \theta_{i} / 2\right)
$$


Calculating the above formula can be obtained

$$
\delta W_{G}=-m g a \sum_{i=1}^{n}\left(n-i+\frac{1}{2}\right) \sin \theta_{i} \delta \theta_{i}
$$

In the water, the steel pipe is effected by the gravity and the buoyancy $\boldsymbol{F}$. If we use $V_{p}$ to represent the total volume of the four pipes, the virtual work of buoyancy is

$$
\delta W_{F}=\rho g V_{p} a \sum_{i=1}^{n}\left(n-i+\frac{1}{2}\right) \sin \theta_{i} \delta \theta_{i}
$$

As shown in Fig 1, the end of the steel pipe by a tensile force $\boldsymbol{P}$, the virtual work of the $\boldsymbol{P}$ in the $\mathrm{x}$-axis direction is

$$
\delta W_{P}^{x}=P \sin \theta_{5} a \sum_{i=1}^{n} \cos \theta_{i} \delta \theta_{i}
$$

The virtual work of the $\boldsymbol{P}$ in the y-axis direction is

$$
\delta W_{P}^{y}=-P \cos \theta_{5} a \sum_{i=1}^{n} \sin \theta_{i} \delta \theta_{i}
$$

According to assumption (1), all steel pipes are rigid, and the steel pipes are hinged. Consequently, all the conditions involved in this problem are in line with the ideal constraints, the sum of the algebra of all virtual works are zero, which we can find the steel pipe inclination.

(1.4), (1.5), (1.6), (1.7) are added, and we can get that the virtual power of the active force in the system is zero. That is

$$
\delta W_{G}+\delta W_{P}^{x}+\delta W_{P}^{y}+\delta W_{F}=0(1.8)
$$

And because the virtual displacement of each pipe are independent changes, so

$$
\tan \theta_{i}=\frac{P \sin \theta_{5}}{\left(n-i+\frac{1}{2}\right)\left(m g-\rho g V_{p}\right)+P \cos \theta_{5}}
$$

We can solve $\theta_{i}$ from equation (1.9)

$$
\theta_{i}=\arctan \frac{P \sin \theta_{5}}{\left(n-i+\frac{1}{2}\right)\left(m g-\rho g V_{p}\right)+P \cos \theta_{5}}
$$

\section{Analysis of barrel force.}

In this article we studied that the barrel length is $1 \mathrm{~m}$, diameter $30 \mathrm{~cm}$. Equipment and barrel total mass is $100 \mathrm{~kg}$. And then we take the barrel as the object of study, analysis of its force, as shown in Fig 3.

Then, all forces are decomposed in the horizontal and vertical directions. According to the theoretical mechanics knowledge, we can pair the steel column equations.

In the horizontal direction

$$
T_{4} \sin \theta_{5}=F_{f}
$$

In the vertical direction

$$
T_{4} \cos \theta_{5}=\rho g\left(V_{\text {ball }}+V_{\text {buoy }}+V_{\text {barrel }}+V_{\text {pipe }}\right)-\left(m_{\text {ball }}+m_{\text {pipe }}+m_{\text {buoy }}+m_{\text {barrel }}\right) g
$$

Where:

$T_{4}$ is the tensile force of the barrel.

$\theta_{5}$ is the angle between the barrel axis and the vertical direction

Based on the above analysis of steel pipes force, we know

$$
F_{f}=P(1.13)
$$

And then, we can solve $\theta_{5}$ from equation (1.12)

$$
\theta_{5}=\arccos \left(\frac{\rho g\left(V_{\text {ball }}+V_{\text {buoy }}+V_{\text {barrel }}+V_{\text {pipe }}\right)-\left(m_{\text {ball }}+m_{\text {pipe }}+m_{\text {buoy }}+m_{\text {barrel }}\right) g}{T_{4}}\right)
$$




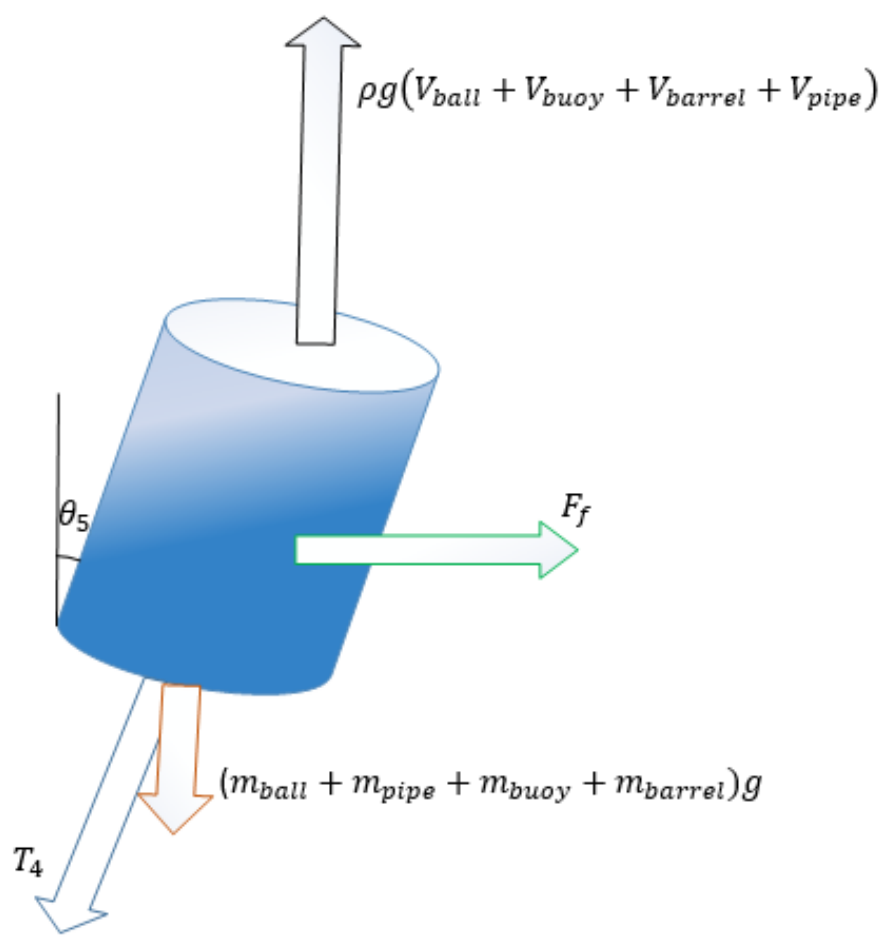

Fig 3. Free-body diagram of barrel

\section{Conclusions}

The weight of the heavy ball studied in this issue is $1200 \mathrm{~kg}$. Now the transmission node is located in the water depth of $18 \mathrm{~m}$, and sea water density of $1.025 \times 10^{3} \mathrm{~kg} / \mathrm{m}^{3}$. In the seawater peaceful conditions, when the wind speed is $12 \mathrm{~m} / \mathrm{s}$, the steel pipe inclination situation as follows

Table 1 . The steel pipe inclination of Wind speed of $12 \mathrm{~m} / \mathrm{s}$

\begin{tabular}{cccc}
\hline$\theta_{1}$ & $\theta_{2}$ & $\theta_{3}$ & $\theta_{4}$ \\
\hline $1.39^{\circ}$ & $1.46^{\circ}$ & $1.63^{\circ}$ & $1.67^{\circ}$ \\
\hline
\end{tabular}

In this condition, the inclination angle $\theta_{5}$ of the barrel is $1.83^{\circ}$.

When the wind speed is $24 \mathrm{~m} / \mathrm{s}$, the steel pipe inclination situation as follows

Table 2. The steel pipe inclination of Wind speed of $24 \mathrm{~m} / \mathrm{s}$

\begin{tabular}{cccc}
\hline$\theta_{1}$ & $\theta_{2}$ & $\theta_{3}$ & $\theta_{4}$ \\
\hline $2.57^{\circ}$ & $2.63^{\circ}$ & $2.67^{\circ}$ & $2.85^{\circ}$ \\
\hline
\end{tabular}

In this condition, the inclination angle $\theta_{5}$ of the barrel is $2.85^{\circ}$.

\section{References}

[1] Lei Wang, Study on Dynamics of Single-Point Mooring systems, D. Ocean University of China., Ocean University of China, 2012.

[2] Junfeng Li,\& Xiong Zhang (2010), Theoretical Mechanics, Beijing, Tsinghua University press.

[3] Fengxiang Mei, On the ideal constraint and the principle of virtual displacement, Mechanics in Engineering, J, 5, 61-63,1999.

[4] Shaoping Wu, Virtual work principle, J. Journal of Higher Correspondence Education. 2000. 